The copyright to this article is held by the Econometric Society, http://www.econometricsociety.org/. It may be downloaded, printed and reproduced only for personal or classroom use. Absolutely no downloading or copying may be done for, or on behalf of, any for-profit commercial firm or other commercial purpose without the explicit permission of the Econometric Society. For this purpose, contact Claire Sashi, General Manager, at sashi@econometricsociety.org. 


\title{
THE CONSUMPTION-BASED CAPITAL ASSET PRICING MODEL
}

\author{
By DARRELl DUfFIE AND William ZAME ${ }^{1}$
}

\begin{abstract}
The paper provides conditions on the primitives of a continuous-time economy under which there exist equilibria obeying the Consumption-Based Capital Asset Pricing Model (CCAPM). The paper also extends the equilibrium characterization of interest rates of Cox, Ingersoll, and Ross (1985) to multi-agent economies. We do not use a Markovian state assumption.
\end{abstract}

KEYwORDS: Finance, general equilibrium theory, asset pricing theory, continuous-time stochastic processes, CAPM.

\section{INTRODUCTION}

THIS WORK PROVIDES sufficient conditions on agents' primitives for the validity of the Consumption-Based Capital Asset Pricing Model (CCAPM) of Breeden (1979). As a necessary condition, Breeden showed that in a continuous-time equilibrium satisfying certain regularity conditions, one can characterize returns on securities as follows. The expected "instantaneous" rate of return on any security in excess of the riskless interest rate (the security's expected excess rate of return) is a multiple common to all securities of the "instantaneous covariance" of this excess return with aggregate consumption increments. This common multiple is the Arrow-Pratt measure of risk aversion of a representative agent. (Rubinstein (1976) published a discrete-time precursor of this result.) The existence of equilibria satisfying Breeden's regularity conditions had been an open issue. We also show that the validity of the CCAPM does not depend on Breeden's assumption of Markov state information, and present a general asset pricing model extending the results of Cox, Ingersoll, and Ross (1985) as well as the discrete-time results of Rubinstein (1976) and Lucas (1978) to a multi-agent environment.

Since the CCAPM was first proposed, much effort has been directed at finding sufficient conditions on the model primitives: the given assets, the agents' preferences, the agents' consumption endowments, and (in a production economy) the feasible production sets. Conditions sufficient for the existence of continuous-time equilibria were shown in Duffie (1986), but the equilibria demonstrated were not shown to satisfy the additional regularity required for the CCAPM. In particular, Breeden assumed that all agents choose pointwise interior consumption rates, in order to characterize asset prices via the first order conditions of the Bellman equation. Interiority was also assumed by Huang (1987) in demonstrating a representative agent characterization of equilibrium, an approach exploited here. The use of dynamic programming and the Bellman equation, aside from the difficulty it imposes in verifying the existence of interior

\footnotetext{
${ }^{1}$ Financial support from the National Science Foundation is gratefully acknowledged. We thank Scott Richard, Kerry Back, Sandy Grossman, Ho-mou Wu, Don Brown, a co-editor, as well as two referees for comments.
} 
optima and sufficiently smooth value functions and feedback controls, tends to emphasize Markovian state information. As we show, the Markov assumption does not actually play a role in the CCAPM pricing relation.

Our paper unfolds in the following way. Section 2 presents a static analogue of our approach, showing that the consumption-based and market-portfolio-based versions of the CAPM are of course the same. Section 3 lays out the model primitives and the definition of a continuous-time equilibrium. Section 4 states the regularity conditions on the primitives of an economy that are used to achieve the representative agent equilibrium pricing model described in Section 5.

The method of proof is roughly as follows. First, we show the existence of an Arrow-Debreu equilibrium for the static infinite-dimensional economy induced by complete forward markets at time zero. Under our assumptions, in particular infinite marginal utility for consumption at zero, we are able to show that every agent's equilibrium consumption choice is pointwise interior. This marginal utility condition, however, prevents us from obtaining a uniform properness condition on preferences, the now standard assumption of Mas-Colell (1986) that has previously been applied in this setting and elsewhere extended. Instead, we show that uniform properness can be avoided in this setting. ${ }^{2}$ Second, following the method of Duffie and Huang (1985), we implement the Arrow-Debreu equilibrium as a stochastic continuous-trading equilibrium, using the continuoustime spanning properties of long-lived securities. Third, we use Huang's (1987) characterization of the equilibrium spot consumption price process as the marginal utility of a representative agent's utility for the aggregate endowment process.

In Section 6, the representative-agent pricing formula is used to prove that the CCAPM applies to the stated equilibrium, and to extend the characterization of real equilibrium interest rates of Cox, Ingersoll, and Ross (1985) to multi-agent economies that are not necessarily Markovian.

\section{THERE'S ONLY ONE CAPM}

For comparison with our continuous-time results, consider a static pure exchange model, with agents defined by endowments $e^{1}, \ldots, e^{m}$ in the space $L$ of random variables with finite variance on a given probability space, and by von Neumann-Morgenstern utility functions $u_{1}, \ldots, u_{m}$ on the real line. There are also securities in zero net supply with payoffs $D=\left(D^{0}, \ldots, D^{N}\right)$ in $L$, where $D^{0}$ is a nonzero constant, say $D^{0} \equiv 1$. An equilibrium is a vector $S=\left(S^{0}, \ldots, S^{N}\right)$ of security prices such that there exist portfolios $\theta^{1}, \ldots, \theta^{m}$ in $\mathbb{R}^{N+1}$ summing to

\footnotetext{
${ }^{2}$ As this paper was being written, we received a manuscript of Araujo and Monteiro (1986), who have independently obtained a similar abstract existence result. Araujo and Monteiro also obtain results for the special case of separable utility functions; these results are somewhat different from ours. More recently, Karatzas, Lakner, Lehoczky, and Shreve (1988) have produced an existence proof for the special case of separable utility functions, using a much different approach that has since been simplified by Dana and Pontier (1989). Karatzas, Lakner, Lehoczky, and Shreve (1988) also provide conditions for uniqueness of complete markets equilibria in our setting.
} 
zero and solving, for respective $i$, the problem

$$
\max _{\boldsymbol{\theta} \in \mathbf{R}^{N+1}} E\left[u_{i}\left(e^{i}+\boldsymbol{\theta} \cdot D\right)\right] \text { subject to } \boldsymbol{\theta} \cdot S=0 .
$$

If, for all $i, u_{i}$ is quadratic and $e^{i} \in \operatorname{span}\left(D^{0}, \ldots, D^{N}\right)$, then the equilibrium allocation is fully Pareto efficient. (For simplicity, we take it that preferences are strictly monotone at the equilibrium allocation so as to neglect the usual satiation problem with quadratic utility.) Thus there are positive weights $\lambda_{1}, \ldots, \lambda_{m}$ such that the von Neumann-Morgenstern function $u_{\lambda}$ defined by

$$
u_{\lambda}(c)=\max _{c_{1}+\cdots+c_{m} \leqslant c} \sum_{i=1}^{m} \lambda_{i} u_{i}\left(c_{i}\right)
$$

defines equilibrium security prices by the representative agent pricing formula

$$
S^{n}=E\left[u_{\lambda}^{\prime}(e) D^{n}\right], \quad 0 \leqslant n \leqslant N,
$$

where $e=e^{1}+\cdots+e^{m}$ is aggregate consumption. It is easy to show that $u_{\lambda}$ is also quadratic, implying that $S^{n}=E\left[(A+B e) D^{n}\right]$ for some numbers $A$ and $B$, which leaves

$$
S^{n}=K_{1} E\left(D^{n}\right)+K_{2} \operatorname{cov}\left(e, D^{n}\right)
$$

for some other coefficients $K_{1}$ and $K_{2}$. If, for any security $n$, the quantities $S^{0}$, $\operatorname{var}(e)$, and $S^{n}$ are all nonzero, simple manipulation reduces this asset pricing model to the classical equation:

$$
E\left(\mathscr{R}^{n}\right)-r=\beta_{\text {en }}\left[E\left(\mathscr{R}^{e}\right)-r\right],
$$

where $\mathscr{R}^{n}=D^{n} / S^{n}$ is the return on asset $n, r=1 / S^{0}$ is the riskless rate of return, $\mathscr{R}^{e}=e /\left(\theta^{e} \cdot S\right)$ is the return on any portfolio $\theta^{e}$ whose total dividend is $\theta^{e} \cdot D=e$ (aggregate consumption), and $\beta_{e n}=\operatorname{cov}\left(\mathscr{R}^{e}, \mathscr{R}^{n}\right) / \operatorname{var}\left(\mathscr{R}^{e}\right)$ is the "beta" of asset $n$ with respect to the portfolio $\theta^{e}$ paying aggregate consumption.

This is the well known Capital Asset Pricing Model (CAPM), presently in a slightly unusual guise. In order to see the equivalence between this version of the CAPM and the traditional (Sharpe (1964)-Lintner (1965)) market-portfolio-based CAPM, we could equally well view the endowment $e^{i}$ of agent $i$ as the payoff $\gamma^{i} \cdot D$ of an endowed portfolio $\gamma^{i} \in \mathbb{R}^{N+1}$ of securities. In this case, the securities are held instead in positive total supply $M=\sum_{i} \gamma^{i} \geqslant 0$. The portfolio $M$ is the market portfolio. Of course, since $M \cdot D=e$, we could take $\theta^{e}$ to be $M$, so that the (above) CAPM is the original CAPM

$$
E\left(\mathscr{R}^{n}\right)-r=\beta_{M n}\left[E\left(\mathscr{R}^{M}\right)-r\right]
$$

in which beta coefficients $\beta_{M n}=\operatorname{cov}\left(\mathscr{R}^{M}, \mathscr{R}^{n}\right) / \operatorname{var}\left(\mathscr{R}^{M}\right)$ are measured with respect to the return $\mathscr{R}^{M}=(M \cdot D) /(M \cdot S)$ on the market portfolio.

\footnotetext{
${ }^{3}$ Although the aggregate portfolio of securities is by definition zero, the fact that each agent's endowment is in the span of the security dividends implies that $e$ is also in their span, implying the existence of a suitable $\theta^{e}$.
} 
As one moves from a single-period to a multi-period setting, the CAPM still applies under suitable conditions, provided one measures betas with respect to current aggregate consumption, rather than the market portfolio. Suppose, for example, that the model has multiple periods and, for simplicity, that there are complete security markets. We can let $\hat{S}^{n}$ denote the market value of the $n$th security after its dividend $D^{n}$ has been realized. The initial price $S^{n}$ of the security reflects the total payoff $D^{n}+\hat{S}^{n}$, and is therefore given by the pricing formula

$$
S^{n}=E\left[u_{\lambda}^{\prime}(e)\left(D^{n}+\hat{S}^{n}\right)\right],
$$

for a suitable vector $\lambda$. The consumption-based CAPM still applies as stated (CCAPM), once the return on security $n$ is redefined as the total return $\mathscr{R}^{n}=\left(D^{n}+\hat{S}^{n}\right) / S^{n}$. The analogous equation (MCAPM), substituting $\mathscr{R}^{M}$ everywhere for $\mathscr{R}^{e}$, is not correct, however, unless by some coincidence $\mathscr{R}^{M}$ and $\mathscr{R}^{e}$ are perfectly correlated, which could be true for example, if endowment "shocks" are independently distributed. Barring extremely special assumptions such as this, it would be a strange quirk indeed if the market-portfolio-based CAPM applies to a multi-period model.

The representative-agent method of proof of the CAPM sketched out above is extended to the continuous-time case in this paper. By adopting a continuous-time formulation, one can drop the quadratic utility assumption, as originally shown by Breeden (1979). The resulting "instantaneous" mean and covariance re-interpretation of the above CAPM is, for practical purposes however, no better than is the closeness of utility to quadratic for consumption over empirical time intervals. By "empirical" time intervals, we mean the time intervals over which data are taken and over which actual consumers do not adjust their rates of consumption expenditures. As Grossman and Laroque (1987) point out, transactions costs reduce the frequency of changes in consumption expenditures, in some cases severely, reducing the quality of the relationship: "marginal utility for actual consumption equals marginal indirect utility for total wealth," the principle element of the consumption-based CAPM.

As Breeden, Gibbons, and Litzenberger (1986) point out, empirical results on the consumption-based CAPM are "mixed." The CAPM is nevertheless a good theoretical starting point. In fact, for additively separable utility, it's the "only game in town" as far as demonstrated equilibrium models of security returns. (For an example with the more general recursive utility formulation, see Epstein and Zin (1989).) The market-based multi-period CAPM does not generally hold, as shown by Merton (1973), and as pointed out again above. It is far from dead, however, as a practical model. Chamberlain (1988) recently directed new theoretical attention toward an intertemporal market-based CAPM, based on an assumed equilibrium satisfying certain properties. The key property Chamberlain assumed, aside from mathematical regularity conditions, is that the marginal utility for aggregate consumption of the representative agent, an analogue to the random variable $u_{\lambda}^{\prime}(e)$, is a (measurable) function of some one-dimensional Brownian motion. In the general setting of multi-dimensional Brownian motion, 
one cannot claim that $u_{\lambda}^{\prime}(e)$ depends only on a single Brownian motion, although that may happen in special cases. For example, if uncertainty is generated only by a one-dimensional Brownian motion, then the market-based CAPM is in principle correct, but tautologically so, since every Ito process is "instantaneously" perfectly correlated with every other Ito process in that case. That is, the " $\theta$-portfolio CAPM" is correct for any portfolio $\theta$, with a one-dimensional Brownian source of uncertainty.

\section{THE CONTINUOUS-TIME ECONOMY}

We quickly lay out the model primitives and the definition of an equilibrium. Background information can be found, for example, in Duffie and Huang (1985) and Duffie (1986).

We work with a finite time interval $[0, T]$, a probability space $(\Omega, \mathscr{F}, P)$ on which is defined a standard Brownian motion $B$ in $\mathbb{R}^{K}$, and the augmented filtration $F=\left\{\mathscr{F}_{t}: t \in[0, T]\right\}$ of sub-tribes ( $\sigma$-algebras) of $\mathscr{F}$ naturally generated by $B$. Unless otherwise indicated, all probabilistic statements are made, suppressing "almost surely," relative to the filtered probability space $(\Omega, \mathscr{F}, F, P)$, our basic model for information and beliefs. At each date $t$ in $[0, T]$, there are markets for securities and a single consumption commodity. Our consumption space is the vector space $L$ of square-integrable predictable stochastic processes. ${ }^{4}$ Informally, if $x \in L$ is a consumption process, then at any time $t$, the consumption rate $x_{t}$ is based only on information available up to that time, and $E\left(\int_{0}^{T} x_{t}^{2} d t\right)<\infty$.

Each of the $m$ agents in this (pure exchange) economy is represented by a pair $\left(U^{i}, e^{i}\right)$, where $U^{i}$ is a utility function on the (usual) positive cone $L_{+}$of consumption processes and $e^{i} \in L_{+}$is an endowment process, for $i \in\{1, \ldots, m\}$.

The remaining primitives are financial securities in zero net supply paying dividends in units of account, just as in Arrow's (1953) original paper on general equilibrium with securities. We designate one of these securities as a numeraire and for simplicity take it to be a pure discount bond redeemable at the terminal date $T$ for one unit of account. This is not a monetary model, but until we later convert prices to the consumption numeraire in order to characterize real security prices, it would be equivalent to treat the numeraire security as inside money. Since the total supply of each security is zero, in equilibrium, no agent is required to "carry away" any of the securities after trading is complete at the terminal date.

The cumulative dividends of the numeraire security are described by the process $D^{0}$ defined by $D_{t}^{0}=0, t<T$, and $D_{T}^{0}=1$. The remaining securities, say $N$ in number, are represented by cumulative dividend processes $D^{1}, \ldots, D^{N}$, where, for each security $n, D^{n}$ is an Ito process such that $D_{T}^{n}$ has finite variance. By Ito

\footnotetext{
${ }^{4}$ Formally, $L=L^{2}(\Omega \times[0, T], \mathscr{P}, \nu)$, where $\mathscr{P}$ is the predictable tribe on $\Omega \times[0, T]$ (that generated by the left-continuous adapted processes) and $\nu$ is the product of $P$ and Lebesgue measure on $[0, T]$ restricted to $\mathscr{P}$.
} 
process, we mean that $D$ satisfies a stochastic differential representation

$$
d D_{t}=\mu_{D}(t) d t+\sigma_{D}(t) d B_{t},
$$

where $\mu_{D}$ is an $N$-dimensional predictable process and $\sigma$ is an $N \times K$-dimensional predictable process. (There is no Markovian assumption; $\mu_{D}(t)$ and $\sigma_{D}(t)$ may depend on the entire past history of the economy.) The entire economy is thus described by a collection

$$
\mathscr{E}=\left((\Omega, \mathscr{F}, F, P),\left(U^{i}, e^{i}\right), D\right), \quad i \in\{1, \ldots, m\},
$$

where $D=\left(D^{0}, \ldots, D^{N}\right)$ is the $(N+1)$-dimensional cumulative dividend process.

Agents take as given a spot consumption price process $p \in L$ and an $(N+1)$ dimensional Ito security price ${ }^{5}$ process $S=\left(S^{0}, \ldots, S^{N}\right)$. The gain process $G=$ $(S+D)$ is therefore an Ito process, and we can write $d G_{t}=\mu_{t} d t+\sigma_{t} d B_{t}$ for some $\mu$ and $\sigma$. This allows one to define cumulative gains from trade for a predictable portfolio process $\theta=\left(\theta^{0}, \ldots, \theta^{N}\right)$. A fixed portfolio $\bar{\theta} \in \mathbb{R}^{N+1}$, for example, generates from some time $\tau$ until some time $s>\tau$ the interim gain $\int_{\tau}^{s} \bar{\theta} d G_{t}=\bar{\theta} \cdot\left[\left(D_{s}-D_{\tau}\right)+\left(S_{s}-S_{\tau}\right)\right]$, the obvious sum of dividend gain and capital gain. For regularity, we demand that a portfolio process $\theta$ be square-integrable, meaning $E\left(\int_{0}^{T} \theta_{t}^{\top} \sigma_{i} \sigma_{t}^{\top} \theta_{t} d t\right)<\infty$, and that the integral $\int \theta_{t} \cdot \mu_{t} d t$ be well-defined, meaning $\int_{0}^{T}\left|\theta_{t} \cdot \mu_{t}\right| d t<\infty$ almost surely. These regularity conditions regarding a portfolio process $\theta$ imply that the gain-from-trade integral $\int \theta_{t} d G_{t}=\sum_{n=0}^{N} \int \theta_{t}^{n} d G_{t}^{n}$ is well-defined, and further that $\int \theta_{t} d G_{t}$ is a martingale whenever $G$ is a martingale. The vector space $\Theta$ of all portfolio processes includes strategies that vary a portfolio continually in time, a trading model inaugurated by Merton (1969).

A budget-feasible plan for agent $i$ is a pair $(x, \theta) \in L_{+} \times \Theta$ such that, for any time $t$,

$$
\theta_{t} \cdot S_{t}=\int_{0}^{t} \theta_{s} d G_{s}+\int_{0}^{t} p_{s}\left(e_{s}^{i}-x_{s}\right) d s,
$$

and such that $\theta_{T}=0$. Relation (2) is merely the accounting restriction that current portfolio wealth must be generated only from trading gains and net consumption purchases. The terminal restriction $\theta_{T}=0$ (no terminal debts) is the essential budget constraint. A budget feasible plan $(x, \theta)$ for agent $i$ is optimal for agent $i$ if there is no budget feasible plan $\left(x^{\prime}, \theta^{\prime}\right)$ such that $U^{i}\left(x^{\prime}\right)>U^{i}(x)$. An equilibrium for an economy $\mathscr{E}$ is a collection $\left((S, p),\left(x^{1}, \theta^{1}\right), \ldots,\left(x^{m}, \theta^{m}\right)\right)$ such that, given the security price process $S$ and the consumption spot price process $p$, for each agent $i$ the plan $\left(x^{i}, \theta^{i}\right)$ is optimal, and markets clear: $\sum_{i} x^{i}-e^{i}=0$ and $\sum_{i} \theta^{i}=0$. Although Duffie (1986) shows conditions on an economy sufficient for the existence of such an equilibrium, those conditions are not sufficient for the asset pricing models of interest here. Appendix $\mathrm{C}$ discusses the issue of nominal versus real (consumption numeraire) primitive dividend

\footnotetext{
${ }^{5}$ We take the convention of cum dividend security prices, so that $S_{T}$, for example, is the market value at time $T$ of any lump sum dividends paid at time $T$.
} 
processes, showing that we can accommodate the latter by more complicated arguments. Just as in Arrow's (1953) paper, however, it is much easier to describe spanning assumptions for purely nominal securities. Duffie (1986) indicates how the assumption of Ito dividend processes can be relaxed.

\section{EQUILIBRIUM CONDITIONS}

We consider the following conditions on an economy.

(A.1) For each agent $i, U^{i}$ is represented by a smooth function $u_{i}: \mathbb{R}_{+} \times[0, T] \rightarrow \mathbb{R}$ in the form

$$
U^{i}(x)=E\left[\int_{0}^{T} u_{i}\left(x_{t}, t\right) d t\right]
$$

where, for each $t$ in $[0, T]$, the function $u_{i}(\cdot, t): \mathbb{R}_{+} \rightarrow \mathbb{R}$ is strictly concave, increasing, with the first derivative on $(0, \infty)$ denoted $u_{i c}(\cdot, t)$ satisfying $\lim _{k \downarrow \downarrow 0} u_{i c}(k, t)=+\infty$.

By smooth, we mean that for any $\varepsilon>0$ sufficiently small, the restriction of $u_{i}$ to $(\varepsilon, \bar{e}+\varepsilon) \times[0, T]$ is $C^{\infty}$, where $\bar{e}$ is the essential supremum of the aggregate consumption process (which might be $+\infty$ ). A $C^{\infty}$ function is one with an extension to an open set having continuous derivatives of any order. ${ }^{6}$ This can be relaxed. ${ }^{7}$ A frequently used example satisfying (A.1) is the function $u(k, t)=$ $\exp (-\gamma t) k^{\alpha}$ for some $\gamma>0$ and $\alpha \in(0,1)$. Lehoczky and Shreve (1986) have a closed form solution for this case, provided agents have the same utility function.

(A.2) The aggregate endowment process $e=\sum_{i=1}^{m} e^{i}$ is an Ito process, bounded away from zero, where the stochastic differential representation $d e_{t}=\mu_{e}(t) d t+$ $\sigma_{e}(t) d B_{t}$ is such that $E\left(\int_{0}^{T} \sigma_{e}(t) \cdot \sigma_{e}(t) d t\right)<\infty$.

Again, there is no Markov assumption here; $\mu_{e}(t)$ and $\sigma_{e}(t)$ can depend generally on the entire history of the economy up to time $t$. The assumption that $e$ is bounded away from zero, however, is restrictive (see Araujo and Monteiro (1987)), and is used to ensure that spot prices for consumption are bounded, given the "infinite marginal utility at zero" assumption in (A.1). With this boundary condition on preferences, aggregate consumption might naturally be bounded away from zero in a production economy with suitable technological possibilities. We have limited ourselves to pure exchange economies for simplicity; in principle, our results should extend to production economies under natural regularity conditions.

The following is a spanning assumption; because of this assumption, in equilibrium any conceivable consumption plan can be financed by some portfolio

\footnotetext{
${ }^{6}$ We later use the fact that continuity of the derivatives and concavity of $u_{i}(\cdot, t)$ imply uniform convergence of the limit indicated in (A.1).

${ }^{7}$ Working through our proofs shows that a $C^{3}$ assumption on $u_{i}$ would more than suffice.
} 
process at some initial cost, and any conceivable additional security is redundant.

(A.3) The martingales $M^{1}, \ldots, M^{N}$ defined by

$$
M_{t}^{n}=E\left[D_{T}^{n} \mid \mathscr{F}_{t}\right], \quad t \in[0, T],
$$

form a martingale generator.

Assumption (A.3) means that any martingale $Y$ can be represented in the form

$$
Y_{t}=Y_{0}+\sum_{n=1}^{N} \int_{0}^{t} \theta_{s}^{n} d M_{s}^{n}
$$

for predictable processes $\theta^{1}, \ldots, \theta^{N}$ stochastically integrable with respect to $M^{1}, \ldots, M^{N}$. Since any martingale such as $M=\left(M^{1}, \ldots, M^{N}\right)$ has a stochastic differential representation of the form $d M_{t}=\varphi_{t} d B_{t}$, where $\varphi$ is a $N \times K$ matrixvalued process, for (A.3) it is necessary and sufficient that the rank of $\varphi$ is $K$ almost everywhere (implying that $N \geqslant K$ ). For a trivial example, let $D^{n}=B^{n}$, $1 \leqslant n \leqslant K$. The "log-normal" case $D_{t}^{n}=\exp \left(B_{t}^{n}\right), 1 \leqslant n \leqslant N$ can also be shown to satisfy (A.3).

Assumption (A.3) can in fact be weakened to an assumption that, under substitution of some probability measure $Q$ uniformly equivalent ${ }^{8}$ to $P$, the martingales defined by (4) form a martingale generator under $Q$. (See Appendix C, or Duffie (1986) for further details.)

\section{A REPRESENTATIVE AGENT EQUILIBRIUM ASSET PRICING MODEL}

A representative agent for a given equilibrium $\left((S, p),\left(x^{1}, \theta^{1}\right), \ldots,\left(x^{m}, \theta^{m}\right)\right)$ for $\mathscr{E}$ is a single agent $\left(U_{\lambda}, e\right)$ having: (a) the same total endowment $e$ of $\mathscr{E}$, (b) a utility function $U_{\lambda}$ defined by

$$
U_{\lambda}(x)=\sup _{\left(x^{1}, \ldots, x^{m}\right) \in L_{+}^{m}} \sum_{i} \lambda_{i} U_{i}\left(x^{i}\right) \text { subject to } \sum_{i} x^{i} \leqslant x,
$$

for some coefficient vector $\lambda \in \mathbb{R}_{+}^{m}$, and (c) the same equilibrium price processes $(S, p)$ (in a no-trade equilibrium) for the single-agent economy $\mathscr{E}_{\lambda}=$ $\left((\Omega, \mathscr{F}, F, P),\left(U_{\lambda}, e\right), D\right)$.

Under preference assumption (A.1), we know that if $U_{\lambda}$ is defined by (5), then

$$
U_{\lambda}(x)=E\left[\int_{0}^{T} u_{\lambda}\left(x_{t}, t\right) d t\right]
$$

where

$$
u_{\lambda}(k, t)=\sup _{\left(k_{1}, \ldots, k_{m}\right) \in \mathbb{R}_{+}^{m}} \sum_{i} \lambda_{i} u_{i}\left(k_{i}, t\right) \quad \text { subject to } \sum_{i} k_{i} \leqslant k .
$$

${ }^{8}$ A probability measure $Q$ is uniformly equivalent to $P$ if the Radon-Nikodym derivative $d Q / d P$ is bounded and bounded away from zero. 
From this fact, it is easy to see that $U_{\lambda}$ also satisfies preference assumption (A.1), using the implicit function theorem to demonstrate smoothness.

Huang (1987) demonstrated a representative agent in this sense for a given Markovian continuous-time equilibrium satisfying certain regularity conditions. Our first result establishes the existence of an equilibrium with the required regularity conditions, and the obvious analogue of the Lucas (1978) "stochastic Euler equation." Later, we extend the result to obtain a more general pricing model. We let $u_{\lambda c}(\cdot, t)$ denote the first derivative of $u_{\lambda}(\cdot, t)$. The following result provides for the existence of an equilibrium $\left((S, p),\left(x^{1}, \theta^{1}\right), \ldots,\left(x^{m}, \theta^{m}\right)\right)$ for $\mathscr{E}$ and characterizes the real (consumption-numeraire) security price process $\hat{S_{t}}=$ $S_{t} / p_{t}$ in terms of the representative agent's marginal utility $u_{\lambda c}\left(e_{t}, t\right)$ for aggregate consumption and in terms of the real dividend process $\hat{D}_{t}$ defined by $d \hat{D}_{t}=p_{t}^{-1} d D_{t}$.

THEOREM 1: Under conditions (A.1)-(A.3) on the economy $\mathscr{E}$, there exists an equilibrium with a representative agent $\left(U_{\lambda}, e\right)$ such that, for any time $t$, the vector $\hat{S}_{t}$ of real security prices satisfies the representative agent pricing formula

$$
\hat{S_{t}}=\frac{1}{u_{\lambda c}\left(e_{t}, t\right)} E\left[\int_{t}^{T} u_{\lambda c}\left(e_{s}, s\right) d \hat{D}_{s} \mid \mathscr{F}_{t}\right], \quad t \in[0, T) .
$$

Moreover, for any agent $i$ with $e^{i} \neq 0$, the equilibrium consumption process $x^{i}$ is bounded away from zero.

Proof: Step A-The Arrow-Debreu Equilibrium: By Appendix Theorem A4, there exists an Arrow-Debreu equilibrium $\left(x^{1}, \ldots, x^{m} ; \pi\right)$ for the complete markets economy $\left(U^{i}, e_{i}\right)$, where the price functional $\pi: L \rightarrow \mathbb{R}$ is represented by some $p \in L_{+}$in the form

$$
\pi(x)=E\left[\int_{0}^{T} p_{t} x_{t} d t\right], \quad x \in L,
$$

with $p$ bounded and $x^{i}$ bounded away from zero for any $i$ with $e^{i} \neq 0$. This is an extension of the work of Mas-Colell (1986), Yannelis and Zame (1986), and Zame (1988) to the case of preferences that are not uniformly proper. (See footnote 1 for related static equilibrium existence results.) Steps A and B of the proof use only Assumption (A.1) plus the assumption from (A.2) that $e$ is bounded away from zero.

Step B-Representative Agent Pricing: We recall the representative agent construction of Huang (1987). Briefly summarizing, by the saddle point theorem, there exist nonnegative constants $\lambda_{1}, \ldots, \lambda_{m}$ (with $\lambda_{i}=0$ if and only if $e^{i}=0$ ) such that the aggregate consumption process $e$ solves the representative agent problem

$$
\max _{x \in L_{+}} E\left[\int_{0}^{T} u_{\lambda}\left(x_{t}, t\right) d t\right] \text { subject to } \pi(x) \leqslant \pi(e) .
$$

Then, by the first order conditions at the solution $e$ to this problem, there exists a 
Lagrange multiplier $\gamma>0$ such that $u_{\lambda_{c}}\left(e_{t}, t\right)=\gamma p_{t}$ almost everywhere. For the details, see Huang (1987). Without loss of generality, $\gamma=1$.

Step C-Dynamic Trading Implementation: In roughly the sense of Duffie and Huang (1985), the static equilibrium $\left(\left(x^{i}\right), \pi\right)$ can be implemented by security trading strategies $\left(\theta^{1}, \ldots, \theta^{m}\right)$ to form a stochastic equilibrium $\left((S, p),\left(x^{i}, \theta^{i}\right)\right)$ with the nominal security pricing formula $S_{t}=E\left[D_{T}-D_{t} \mid \mathscr{F}_{t}\right]$.

To outline the basic parts of this implementation procedure, pick any agent $i$ and let $Y$ be the martingale defined by

$$
Y_{t}=E\left(\int_{0}^{T} p_{s}\left(x_{s}^{i}-e_{s}^{i}\right) d s \mid \mathscr{F}_{t}\right) .
$$

The gain process $G=S+D$ is a martingale generator by the spanning assumption (A.3). By the definition of a martingale generator, there exist predictable processes $\varphi^{1}, \ldots, \varphi^{K}$ such that

$$
Y_{t}=Y_{0}+\sum_{k=1}^{K} \int_{0}^{t} \varphi_{s}^{k} d G_{s}^{k}, \quad t \in[0, T]
$$

Let $\varphi^{0}$ be the predictable process defined by

$$
\varphi_{t}^{0}=Y_{t}-\int_{0}^{t} p_{s}\left(x_{s}^{i}-e_{s}^{i}\right) d s-\sum_{k=1}^{K} \varphi_{t}^{k} S_{t}^{k} .
$$

An exercise shows that the trading strategy $\theta^{i}=\left(\varphi^{0}, \varphi^{1}, \ldots, \varphi^{K}\right)$ forms a budget feasible plan $\left(x^{i}, \theta^{i}\right)$ for agent $i$. A simple proof by contradiction suffices to show that $\left(x^{i}, \theta^{i}\right)$ is moreover optimal for agent $i$. We can choose such a plan for each of the $m$ agents. Now, suppose we replace the trading strategy $\theta^{m}$ so chosen for agent number $m$ with the new trading strategy $\hat{\theta}^{m}=-\sum_{i=1}^{m-1} \theta^{i}$. This implies clearing in the security markets. Consumption market clearing in the given equilibrium for the static economy (as well as the linearity of integration) then implies that $\left(\hat{\theta}^{m}, c^{m}\right)$ is budget feasible for agent $m$. Since $\left(\theta^{m}, c^{m}\right)$ is optimal for $m$, it follows that $\left(\hat{\theta}^{m}, c^{m}\right)$ is also optimal for $m$. Thus we have shown the existence of an equilibrium in the stochastic economy.

Step D-Renormalization of Prices: Using the definition of the consumptionnumeraire prices and dividends, $(\hat{S}, \hat{D})$, given before the statement of the Theorem, and the fact that $p_{t}=u_{\lambda c}\left(e_{t}, t\right)$, we recover the security pricing formula (6).

Q.E.D.

By taking any two times $t$ and $\tau \geqslant t$, we can substitute the expression for $\hat{S}_{\tau}$ given by $(6)$ into the corresponding equation for $\hat{S}_{t}$ to verify the analogue to Lucas' (1978) "stochastic Euler equation,"

$$
\hat{S_{t}}=\frac{1}{u_{\lambda c}\left(e_{t}, t\right)} E\left[\int_{t}^{\tau} u_{\lambda c}\left(e_{s}, s\right) d \hat{D}_{s}+u_{\lambda c}\left(e_{\tau}, \tau\right) \hat{S}_{\tau} \mid \mathscr{F}_{t}\right] .
$$

It is convenient (and useful for purposes of generality) to consider more general forms of security dividends. To begin, we consider a cumulative real 
dividend process in the form of an Ito process $Y$ having a final value $Y_{T}$ with finite variance. By definition, $Y$ solves a stochastic differential equation of the form

$$
d Y_{t}=\mu_{Y}(T) d t+\sigma_{Y}(t) d B_{t},
$$

where $\mu_{Y}$ and $\sigma_{Y}$ are (possibly path-dependent) processes. (We take $\mu_{Y}$ and $\sigma_{Y}$ to be predictable processes, real-valued and $\mathbb{R}^{K}$-valued, respectively.)

Introducing the new security $Y$ into the model changes nothing in equilibrium, given the spanning assumption (A.3), provided the new security is priced consistently with equation (6), that is, provided the current real price of the security is

$$
S_{t}^{Y}=\frac{1}{u_{\lambda c}\left(e_{t}, t\right)} E\left[\int_{t}^{T} u_{\lambda c}\left(e_{s}, s\right) d Y_{s} \mid \mathscr{F}_{t}\right] .
$$

(Since the security is redundant, any other price process allows arbitrage.) Likewise, any additional security paying a terminal lump sum dividend $\delta$ is also redundant, and at time $t$ has the equilibrium real price $E\left[u_{\lambda c}\left(e_{T}, T\right) \delta \mid \mathscr{F}_{t}\right] /$ $u_{\lambda c}\left(e_{t}, t\right)$. (We only assume that $\delta$ is $\mathscr{F}_{T}$-measurable and of finite variance.) By additivity, the equilibrium price process $S^{Y \delta}$ for a security promising the Ito dividend process $Y$ as well as a terminal dividend $\delta$ is therefore defined by

$$
S_{t}^{Y \delta}=\frac{1}{u_{\lambda c}\left(e_{t}, t\right)} E\left[\int_{t}^{T} u_{\lambda c}\left(e_{s}, s\right) d Y_{s}+u_{\lambda c}\left(e_{T}, T\right) \delta \mid \mathscr{F}_{t}\right], \quad t \in[0, T] .
$$

This is formalized by the following corollary to Theorem 1 for the augmented economy $\mathscr{E}^{\text {Y⿱ }}$ defined by the original economy $\left.\mathscr{E}=\left((\Omega, \mathscr{F}, F, P),\left(U^{i}, e^{i}\right), D\right)\right)$ and the additional security paying the real dividend process $Y$ as well as the terminal real dividend $\delta$. (The result extends to the augmentation of any number of additional securities.)

COROLlARY: Further to the statement of Theorem 1, for any cumulative (Ito) dividend process $Y$ of finite variance and any terminal lump sum dividend $\delta$ of finite variance, the augmented economy $\mathscr{E}^{Y \delta}$ has an equilibrium with the same consumption allocation and the same representative agent in which the real price process $S^{Y \delta}$ for the security $(Y, \delta)$ satisfies equation ( 7$)$.

Intermediate lump sum dividends paid at (possibly random stopping) times can also be priced in the obvious way, as shown in Duffie (1986).

\section{THE CCAPM}

We will now characterize the equilibrium excess rate of return on any security paying a cumulative (Ito) real dividend process $Y$ plus some lump sum terminal dividend $\delta$. For notational convenience, we let $V$ denote the equilibrium real price process $S^{Y \delta}$ of this security, as defined by (7). 
Consider the process $Z$ defined by $Z_{t}=\int_{0}^{t} p_{s} d Y_{s}+p_{t} V_{t}$. From (7), it is easy to check that $Z$ is a martingale. Since $V$ is an Ito process, we can always write

$$
d V_{t}=\mu_{V}(t) d t+\sigma_{V}(t) d B_{t},
$$

for some predictable process $\mu_{V}$ and $\mathbb{R}^{K}$-valued predictable process $\sigma_{V}$. Also, by Ito's Lemma (as stated in Appendix B),

$$
\begin{aligned}
d Z_{t}= & {\left[p_{t} \mu_{Y}(t)+p_{t} \mu_{V}(t)+V_{t} \mu_{p}(t)+u_{\lambda c c}\left(e_{t}, t\right) \sigma_{e}(t) \cdot \sigma_{V}(t)\right] d t } \\
& +\sigma_{Z}(t) d B_{t},
\end{aligned}
$$

for some process $\sigma_{Z}$ that we need not calculate. Since $Z$ is a martingale, for almost every time $t$,

$$
p_{t}\left[\mu_{Y}(t)+\mu_{V}(t)\right]+V_{t} \mu_{p}(t)+u_{\lambda c c}\left(e_{t}, t\right) \sigma_{e}(t) \cdot \sigma_{V}(t)=0 .
$$

Dividing through by $p_{t}=u_{\lambda c}\left(e_{t}, t\right)$, which is everywhere nonzero, we have the basic rate of return relationship

$$
\mu_{V}(t)+\mu_{Y}(t)-r_{t} V_{t}=\frac{-u_{\lambda c c}\left(e_{t}, t\right)}{u_{\lambda c}\left(e_{t}, t\right)} \sigma_{V}(t) \cdot \sigma_{e}(t),
$$

where $r_{t}=-\mu_{p}(t) / p_{t}$. For $V_{t} \neq 0$, we can define the total real rate of expected return on this security at time $t$ as

$$
\overline{\mathscr{R}}_{t}=\frac{\left[\mu_{V}(t)+\mu_{Y}(t)\right]}{V(t)},
$$

the expected real rate of capital plus dividend gain divided by current market value. Letting $\sigma_{\mathscr{R}}(t)=\sigma_{V}(t) / V(t)$, it is common to treat $\sigma_{\mathscr{R}}(t) \cdot \sigma_{\mathscr{R}}(t)$ as the "instantaneous variance of return" of the security. (After completing the statement of the CCAPM, however, we make a critical remark concerning the generality of this definition.) We now have the more familiar CCAPM relation

$$
\overline{\mathscr{R}}_{t}-r_{t}=\frac{-u_{\lambda c c}\left(e_{t}, t\right)}{u_{\lambda c}\left(e_{t}, t\right)} \sigma_{\mathscr{R}}(t) \cdot \sigma_{e}(t),
$$

taking the common interpretation of $\sigma_{\mathscr{R}}(t) \cdot \sigma_{e}(T)$ as the instantaneous conditional covariance between increments of aggregate consumption process $e$ and the total real return on the given security. The security in question is riskless (in real terms) if $\sigma_{\mathscr{R}}(t) \equiv 0$. Equation (9) then gives us a characterization of the riskless rate of return, $r_{t}=-\mu_{p}(t) / p_{t}$. Since $p_{t}=u_{\lambda c}\left(e_{t}, t\right)$, the riskless rate of return or equivalently, the real equilibrium short term interest rate, is minus the expected rate of growth of marginal utility for consumption of the representative agent. In a more restrictive setting of a single agent and a Markovian state process, Cox, Ingersoll, and Ross (1985) have previously given this characterization of the riskless rate.

More formally, we have the following version of the CCAPM.

Proposition 1 (CCAPM): There exist equilibria for economies satisfying Assumptions (A.1)-(A.3). In equilibrium, at almost every time $t$, the riskless real rate 
of return is $r_{t}=-\mu_{p}(t) / p_{t}$ (minus the expected rate of change of marginal utility for consumption of the representative agent), and the expected real rate of return $\overline{\mathscr{R}}_{t}$ of any security satisfies (9), provided the market value of the security is not zero.

To describe $\sigma_{\mathscr{R}} \cdot \sigma_{\mathscr{R}}$ as the "instantaneous variance of the return of the security" is quite misleading unless the security $Y$, defined by $d Y_{t}=\mu_{Y}(t) d t+$ $\sigma_{Y}(t) d B_{t}$, has $\sigma_{Y}(t)=0$. That is, if the security's dividends are "instantaneously riskless," as is said, then the nomenclature is appropriate. Otherwise, $\sigma_{\mathscr{R}}$ actually corresponds to the "instantaneous variance" of capital returns only, and does not include the effects of the "local risk" of dividend returns.

One can also give the usual "beta" interpretation of relation (9) as follows. Consider any security whose equilibrium real price process $V^{e}$ has a diffusion $\sigma_{V^{e}}$ such that $\sigma_{V^{e}}(t) / V_{t}^{e}=\sigma_{e}(t)$ for all $t$. One can always design such a security by our spanning assumption (A.3). Since the excess expected real rate of return of this security, denoted $\overline{\mathscr{R}}_{t}^{e}$, satisfies the CCAPM (9), we have

$$
\overline{\mathscr{R}}_{t}^{e}-r_{t}=\frac{-u_{\lambda c c}\left(e_{t}, t\right)}{u_{\lambda c}\left(e_{t}, t\right)} \sigma_{e}(t) \cdot \sigma_{e}(t) .
$$

Combining (9) and (10), we have the traditional beta relationship

$$
\mathscr{R}_{t}-r_{t}=\beta_{\mathscr{R}}(t)\left(\mathscr{R}_{t}^{e}-r_{t}\right) \text {, }
$$

where

$$
\beta_{\mathscr{R}}(t)=\frac{\sigma_{\mathscr{R}}(t) \cdot \sigma_{e}(t)}{\sigma_{e}(t) \cdot \sigma_{e}(t)},
$$

assuming the denominator is nonzero.

Relationship (11) is not equivalent to (9), since (11) is true even when (9) is adjusted by replacing the representative agent risk aversion measure $-u_{\lambda c c}\left(e_{t}, t\right) / u_{\lambda c}\left(e_{t}, t\right)$ with any well-defined process. That is, relation (11) states merely that excess expected returns are proportional to consumption betas, while relation (9) also specifies the constant of proportionality. Breeden's original work suggests that (11) will in fact hold without spanning in an appropriately regular equilibrium, using a suitable definition of $\mathscr{R}_{t}^{e}$ as the excess expected return of a particular portfolio with "returns most highly correlated with aggregate consumption increments." Existence of nontrivial continuous-time equilibria with incomplete markets is an open issue. Certainly, spanning is not an issue in a single-agent economy, and (11) can easily be shown to hold in Breeden's sense in a single-agent economy, Markov or not, following the calculations made here, since the equilibrium allocation in a single-agent economy is Pareto optimal. Aside from the single-agent case, or except by a strange coincidence, incomplete markets negates both Pareto optimality and a representative agent.

\section{CONCLUDING REMARKS}

We remark on several extensions of the theory in this paper. As pointed out by Breeden (1979), the CCAPM extends, with limitations, to the case of multiple 
commodities. Back (1988) has given a partial extension to more general information structures than that generated by Brownian motion. Of course, our main result (Theorem 1) and the representative agent asset pricing model (6) apply to an arbitrary information filtration satisfying the usual conditions, provided the integral in equation (6) is well-defined. It is only when we derive the CCAPM itself that we turn to the "instantaneous covariance" characterization of asset returns, which depends on a Brownian information structure. Our abstract existence results in Appendix A also permit an extension of Theorem 1 to the case of multiple commodities and an additive state-dependent utility function $u_{i}$ : $\Omega \times[0, T] \times \mathbb{R}^{\prime} \rightarrow \mathbb{R}$, subject to technical conditions. Of course, the CCAPM is not true with state-dependent utility.

Finally, we remark that our results meet Huang's (1987) sufficient conditions for Markovian equilibrium. That is, if $e$ and $D$ are well behaved functions of a Markov state process $X$, then the equilibrium (real) security prices defined by (6) are also measurable functions of the state process $X$.

\section{Graduate School of Business, Stanford University, Stanford, CA 94305, U.S.A. and}

Department of Mathematics, State University of New York, Buffalo, NY, 14214, U.S.A.

Manuscript received May, 1987; final revision received March, 1989.

\section{APPENDIX A: THE STATIC ARROW-DEBREU ECONOMY}

This Appendix presents the required existence result for the static Arrow-Debreu economy. The precise result we require is rather special, but the underlying result is rather general. Since this underlying result seems potentially useful in other contexts, we digress slightly to present it first.

\section{Abstract Arrow-Debreu Existence Result}

Let $K$ be a normed vector lattice with positive cone $K_{+}$(see Schaefer (1974)). We consider an Arrow-Debreu (pure exchange) economy $\mathscr{E}=\left(\succ_{i}, e^{i}\right)$ in $K$, with $m$ agents described by preference relations $\succeq_{i}$ on $K_{+}$and initial endowments $e^{i} \in K_{+}$. Throughout, we make the usual assumptions on preferences: each $\succeq_{i}$ is reflexive, transitive, complete, convex, continuous, and strictly monotone.

We say that a (Hausdorff) topology $\tau$ on $K$ is compatible if it is weaker than the norm topology and if all order intervals $[0, x]=\{y \in K: 0 \leqslant y \leqslant x\}$ are $\tau$-compact. We say that the preference relation $\succeq$ satisfies the forward cone condition at $x \in K_{+}$if:

$(F C):$ There is $a v \in K_{+}$, an $\varepsilon>0$ and $a \rho>0$ such that $x+\lambda v-z>x$ whenever $x+\lambda v-z \in K_{+}$, $0<\lambda<\rho$, and $\|z\|<\lambda \varepsilon$.

The forward cone condition was introduced by Yannelis and Zame (1986), and is a variant of the backward cone condition ("properness") introduced by Mas-Colell (1986). The validity of the uniform version of the forward cone condition (that is, that $(F C)$ holds at each $x \in K_{+}$, with $v, \varepsilon, \rho$ fixed) is equivalent to uniform properness, which was used by Mas-Colell to obtain the existence of competitive equilibria. However, the pointwise versions of the forward cone condition (which we use) and of properness are incomparable.

An allocation $\left(x^{1}, \ldots, x^{m}\right) \in\left(K_{+}\right)^{m}$ is feasible for $\mathscr{E}$ if $\sum_{l}\left(e^{i}-x^{i}\right)=0$. A competitive equilibrium is a feasible allocation $\left(x^{1}, \ldots, x^{m}\right)$ and a linear functional $\pi$ on $K$ such that, for all $i, \pi \cdot x^{i} \leqslant \pi \cdot e^{i}$ and $x^{i} \geq_{l} y^{i}$ for any $y^{i} \in K_{+}$with $\pi \cdot y^{i} \leqslant \pi \cdot e^{i}$. A feasible allocation $\left(x^{1}, \ldots, x^{m}\right)$ is in the core 
if there exists no other allocation $\left(y^{1}, \ldots, y^{m}\right)$ and nonempty subset $\mathscr{A}$ of agents such that $\sum_{i \in \mathscr{A}}\left(e^{i}-y^{l}\right)=0$ and $y^{l} \succ_{1} x^{i}$ for all $i \in \mathscr{A}$.

Our abstract existence result is:

THEOREM A1: Let $K$ be a normed lattice, let $\tau$ be a compatible topology on $K$, and let $\mathscr{E}$ be an Arrow-Debreu economy in $K$. Assume that: (a) each of the preference relations $\succeq_{1}$ is $\tau$-upper semicontinuous; $(b)$ for each core allocation $\left(x^{1}, \ldots, x^{m}\right)$ for $\mathscr{E}$, each $\succeq$, satisfies the forward cone condition at $x^{l} ;$ and $(c) e=\sum_{l} e^{l}$ is strictly positive. Then $\mathscr{E}$ has a competitive equilibrium $\left(x^{1}, \ldots, x^{m} ; \pi\right)$, where $\pi$ is a positive continuous linear functional on $K$.

To prove Theorem A1, we first consider a restricted economy $\tilde{\mathscr{E}}$. Let $K(e)$ be the order ideal

$$
K(e)=\left\{x \in K: \exists s \in \mathbb{R}_{+}:|x| \leqslant s e\right\},
$$

where $|x|=x^{+}+x^{-}$, and define a norm $\|\cdot\|_{e}$ on $K(e)$ by

$$
\|x\|_{e}=\inf \{s \geqslant 0:|x| \leqslant s e\} .
$$

It is easily checked that, equipped with this norm, $K(e)$ is a normed vector lattice. The economy $\tilde{\mathscr{E}}$ is simply the restriction of $\mathscr{E}$ to $K(e)$. That is, $\mathscr{E}$ has the same agents $\{1, \ldots, m\}$, with the same endowments $e_{1}, \ldots, e_{m}$, and with the preferences obtained by restricting $\succeq_{1}$ to $K(e)$.

The following lemma and its proof are slight variations of those in Zame (1987) as well as Aliprantis, Brown, and Burkinshaw (1987a). The general idea of working on order ideals of the consumption space, allowing one to naturally extend Bewley's (1972) approach, can also be found in Brown (1983) and in Aliprantis, Brown, and Burkinshaw (1987b).

LEMMA A2: The economy $\tilde{\mathscr{E}}$ has a competitive equilibrium $\left(x^{1}, \ldots, x^{m} ; \bar{\pi}\right)$, where $\tilde{\pi}$ is a positive linear functional on $K(e)$ which is continuous with respect to the norm $\|\cdot\|_{e} \cdot-$

Proof: Let $\tilde{\tau}$ denote the restriction of $\tau$ to $K(e)$. The feasible consumption sets for $\tilde{\mathscr{E}}$ are the order ideal $[0, e]$, which is $\tau$-compact (by assumption). Moreover, the endowment $e$ lies in the interior of the positive cone of $K(e)$. It follows ${ }^{9}$ from Theorem 2 of Zame (1986) that $\mathscr{E}$ has a quasi-equilibrium $^{10}\left(x^{1}, \ldots, x^{m} ; \tilde{\pi}\right)$, where $\tilde{\pi}$ is continuous with respect to the norm $\|\cdot\|_{e}$. Strict monotonicity of preferences implies as usual that $\tilde{\pi}$ is positive and that $\left(x^{1}, \ldots, x^{m} ; \tilde{\pi}\right)$ is an equilibrium. Q.E.D.

LEMMA A3: If $\left(x^{1}, \ldots, x^{m} ; \tilde{\pi}\right)$ is an equilibrium for $\tilde{\mathscr{E}}$ and if each of the preference relations $\succeq_{l}$ satisfies the forward cone condition at $x^{t}$, then $\tilde{\pi}$ is continuous with respect to the original norm $\|\cdot\|$ on $K(e)$.

Proof: $:^{11}$ Let $v_{l}, \varepsilon_{i}$, and $\rho_{i}$ be as in the forward cone condition for $\succeq_{i}$ at $x^{t}$. At the cost of replacing $\varepsilon_{t}$ by $\varepsilon_{l} / 2$, we may replace $v_{l}$ with any vector $v_{l}^{\prime} \in K_{+}$such that $\left\|v_{t}-v_{l}^{\prime}\right\|<\varepsilon_{l} / 2$. Since $K(e)$ is dense in $K$, we may without loss of generality assume that $v_{i} \in K(e)$ for each $i$. Set $v=\sum_{l} v_{t}$ and $\varepsilon=\min \left\{\varepsilon_{1}, \ldots, \varepsilon_{m}\right\}$. We claim that

$$
|\tilde{\pi} \cdot y| \leqslant \frac{\tilde{\pi} \cdot v}{\varepsilon}\|y\|, \quad y \in K(e) .
$$

Note first that (since $\tilde{\pi}$ is positive) it suffices to establish this for each $y \in K(e)_{+}$with $\|y\| \leqslant 1$. Suppose that $\tilde{\pi} \cdot y>\tilde{\pi} \cdot v / \varepsilon$. For $\lambda>0$ small enough, $\lambda y \leqslant e$, so $e-\lambda y \in K(e)_{+}$. Since $e=\sum_{l} e^{i}=$ $\sum_{l} x^{i}$, the Riesz Decomposition Property (Schaefer (1974)) allows us to find vectors $z_{l} \in K(e)$ such

${ }^{9}$ Theorem 2 of Zame (1986) assumes that each of the endowments $e^{i}$ lies in the interior of the consumption sets, but this assumption is used only to obtain an equilibrium from a quasi-equilibrium (no monotonicity assumption is used there).

${ }^{10} \mathrm{~A}$ quasi-equilibrium is the same as an equilibrium, except for the substitution of the condition $U\left(x^{\prime}\right)>U\left(x^{l}\right) \Rightarrow \pi \cdot x^{\prime} \geqslant \pi \cdot x^{l}$ in place of the usual optimality condition.

${ }_{11}$ This is the argument of the Price Lemma of Yannelis and Zame (1986). 
that $0 \leqslant z_{l} \leqslant x^{l}$ and $\varepsilon \lambda y=\sum_{i} z_{i}$. Then

$$
e+\lambda v-\varepsilon \lambda y=\sum_{i}\left(x^{i}+\lambda v_{i}-z_{i}\right) \text {. }
$$

Since $\sum_{i} z_{i}=\varepsilon \lambda y$, it follows that $\left\|z_{i}\right\| \leqslant \varepsilon_{i}\|\lambda y\|$ for each $i$, and hence (by the forward cone condition) that $x^{i}+\lambda v_{l}-z_{i} \succ_{i} x^{i}$ for each $i$. On the other hand, $\tilde{\pi} \cdot(\lambda v-\varepsilon \lambda y)<0$ so $\tilde{\pi} \cdot\left(x^{i}+\lambda v_{l}-z_{i}\right)<\tilde{\pi} \cdot x^{i}$ for some $i$. This contradicts the equilibrium properties of $\left(x^{1}, \ldots, x^{m} ; \tilde{\pi}\right)$, so we conclude that $\tilde{\pi} \cdot y \leqslant(\tilde{\pi} \cdot v / \varepsilon)\|y\|$ for each $y \in K(e)_{+}$, and hence that $\tilde{\pi}$ is continuous with respect to the $\|\cdot\|$-norm on $K(e)$, as asserted.

Q.E.D.

Proof (of Theorem A1): By Lemma A2, the economy $\tilde{\mathscr{E}}$ has an equilibrium $\left(x^{1}, \ldots, x^{m} ; \tilde{\pi}\right)$. The equilibrium allocation $\left(x^{1}, \ldots, x^{m}\right)$ is in the core of $\tilde{\mathscr{E}} ;$ since all feasible allocations for $\mathscr{E}$ are actually in $K(e)^{m}$, the allocation $\left(x^{1}, \ldots, x^{m}\right)$ is in the core of $\mathscr{E}$. Since $K(e)$ is dense in $K$, the continuity of $\tilde{\pi}$ (Lemma A3) implies that $\tilde{\pi}$ has a (unique) extension to a positive, continuous linear functional $\pi$ on $K$. Continuity of preferences implies that $\left(x^{1}, \ldots, x^{m} ; \pi\right)$ is an equilibrium for $\mathscr{E}$.

Q.E.D.

An Application to the $L^{2}$ Case with Additive-Separable Preferences

We turn now to the precise result required. From now on, the commodity space $L=L^{2}(\Omega \times$ $[0, T], \mathscr{P}, \nu)$. Let $\mathscr{E}=\left(\succeq_{l}, e^{i}\right)$ denote the corresponding Arrow-Debreu complete markets economy on $L$, where $\succeq$, is represented by a utility function $U^{\prime}$.

THEOREM A4: Under condition (A.1) of Section 3 and the assumption that the aggregate endowment process $e$ is bounded away from zero, $\mathscr{E}$ has a competitive equilibrium $\left(x^{1}, \ldots, x^{m} ; \pi\right)$, where $\pi$ is represented by $p \in L_{+}$as in relation (6) with $p$ bounded and, for all $i$, if $e^{i} \neq 0$, then $x^{i}$ is bounded away from zero.

Proof: Let $K$ denote $L$ equipped with the norm $\|\cdot\|_{1}$ given by

$$
\|x\|_{1}=E\left(\int_{0}^{T}\left|x_{t}\right| d t\right) .
$$

As such, $K$ is a normed vector lattice. It is easily checked that each of the preference relations is continuous in the $\|\cdot\|_{1}$-topology. Let $\tau$ denote the weak topology on $K$ with respect to the topological dual $K^{*}$ of $I$. This topology $\tau$ is compatible. ${ }^{12}$ Moreover, since norm-closed convex sets are weakly closed, the preference relations $\succ_{i}$ are $\tau$-upper semicontinuous.

Fix a measurable (predictable) subset $A$ of $\Omega \times[0, T]$ and a real number $a>0$. If $y \in K_{+}$and $y \geqslant a$ on $A$, then together with the Mean Value Theorem our assumptions imply that

$$
U^{i}(y)-U^{i}(y-z) \leqslant E\left(\int_{0}^{T} z_{t} d t\right) \sup _{t \in[0, T]} u_{i c}(a / 2, t)
$$

whenever $z \in K_{+}$is any vector in $K_{+}$supported on $A$ and bounded above by $a / 2$. Similarly, for any set $H \in \mathscr{P}, h \geqslant 0$, and $y \in K_{+}$with $y \leqslant h$ on $H$, we obtain

$$
U^{i}\left(y+\lambda 1_{H}\right)-U^{i}(y) \geqslant \lambda \nu(H) \inf _{t \in[0, T]} u_{i c}(3 h / 2, t)
$$

whenever $0 \leqslant \lambda \leqslant h$. There is no loss of generality in assuming that each $e^{l}$ is nonzero, and hence that $U^{i}\left(e^{i}\right)>U^{i}(0)$ for each $i$. Suppose $\left(x^{1}, \ldots, x^{m}\right)$ is a core allocation for $\mathscr{E}$. Since $U^{i}\left(x^{i}\right) \geqslant U^{i}\left(e^{i}\right)>$ $U^{i}(0)$, there is a set $A_{i} \in \mathscr{P}$ with $\nu\left(A_{i}\right)>0$ and a number $a_{i}>0$ with $x^{i} \geqslant a_{i}$ on $A_{i}$. If some $x^{j}$ is not bounded away from zero, we can find for each $h>0$ some $H \in \mathscr{P}$ such that $\nu(H)>0$ and $x^{j} \leqslant h$ on $H$. Since $e$ is bounded away from zero, say $e \geqslant c>0$ for some $c$, we can assume without loss that $x^{l} \geqslant c / m$ on $H$ for some agent $l$. Applying (13) and (14) and our assumption that $u_{i c}(k, t) \rightarrow \infty$ as $k \rightarrow 0$, we conclude that agents $j$ and $l$ could exchange appropriate multiples of the commodity bundles $1_{H}$ and $1_{A}$ to effect a Pareto improvement provided $h$ is small enough. Since this would be a contradiction, we conclude that each $x^{j}$ is indeed bounded away from zero.

${ }^{12}$ On order intervals, this topology coincides with the weak topology on $L$ with respect to $L^{*}$; Alaoglu's Theorem implies that order intervals are compact in this topology (Schaefer (1974)). 
We now assert that the forward cone conditions hold at the points $x^{1}, \ldots, x^{m}$. Since the $x^{l}$ 's are all bounded below away from zero and bounded above on some set $C \in \mathscr{P}$ with $\nu(C)>0$, this follows immediately from (13) and (14) (taking $v_{i}=1_{C}$ for each $i$ ). We now conclude from Theorem A1 that $\mathscr{E}$ has an equilibrium $\left(x^{1}, \ldots, x^{m} ; \pi\right)$. Since $\left(x^{1}, \ldots, x^{m}\right)$ is a core allocation, $x^{i}$ is bounded away from zero for each $i$. Moreover, since $\pi$ is $\|\cdot\|_{1}$-continuous, the Riesz representation theorem implies that $\pi$ is represented by a positive bounded function $p$; since $\nu$ is a finite measure, $p$ is in $L$.

Q.E.D.

\section{APPENDIX B: ITO'S LEMMA}

We have used Ito's Lemma repeatedly, and it may be best to state it formally for the record in the setting of Ito processes. If $d X_{t}=\mu_{X}(t) d t+\sigma_{X}(t) d B_{t}$ is an $\mathbb{R}^{n}$-valued Ito process for some $n$, and $f: \mathbb{R}^{n} \times[0, T] \rightarrow \mathbb{R}$ is $C^{2}$ (when extended to an open set), then the process $Y$ defined by $Y_{t}=f\left(X_{t}, t\right)$ is also an Ito process with stochastic differential $d Y_{t}=\mu_{Y}(t) d t+\sigma_{Y}(t) d B_{t}$ defined by $\sigma_{Y}(t)=$ $f_{X}\left(X_{t}, t\right) \sigma_{X}(t)$ and

$$
\mu_{Y}(t)=f_{X}\left(X_{t}, t\right) \mu_{X}(t)+f_{t}\left(X_{t}, t\right)+\frac{1}{2} \operatorname{trace}\left[\sigma_{X}(t)^{\top} f_{X X}\left(X_{t}, t\right) \sigma_{X}(t)\right]
$$

where the vector $f_{X}\left(X_{t}, t\right)$ and matrix $f_{X X}\left(X_{t}, t\right)$ denote the first and second partial derivatives of $f$ with respect to the $X$ arguments. (The same formula applies under weaker conditions.) We actually use two special cases of this result in the paper. One is the case of $n=1, X_{t}=e_{t}$, and $Y_{t}=f\left(X_{t}, t\right)=$ $u_{\lambda c}\left(e_{t}, t\right)$. In this case, $\sigma_{Y}(t)=u_{\lambda c c}\left(e_{t}, t\right) \sigma_{e}(t)$. The second application of Ito's Lemma in the body of the paper is the case of $n=2, \stackrel{X_{t}^{(1)}}{=} p_{t}, X_{t}^{(2)}=V_{t}$, and $Y_{t}=f\left(X_{t}, t\right)=X_{t}^{(1)} X_{t}^{(2)}$. In the latter case, application of Ito's Lemma leads to

$$
\mu_{Y}(t)=p_{t} \mu_{V}(t)+V_{t} \mu_{p}(t)+\sigma_{V}(t) \cdot \sigma_{p}(t)
$$

Details and extensions can be found, for example, in Lipster and Shiryayev (1977).

\section{APPENDIX C: SPANNING WITH REAL PRIMITIVE SECURITY DIVIDENDS}

Just as with Arrow's (1953) model of security markets, we have found it convenient in this paper to define the primitive, exogenously given, security dividends in (nominal) unit of account, not in (real) consumption numeraire terms. This allows an easy development of spanning in continuous-time, based on primitive assumptions on the nominal dividend process $D$. In a general multi-commodity model, there is little alternative, as shown by Hart (1975), since equilibria need not exist in general. (Although Hart worked in a discrete-time setting, the fact that the span of markets may collapse discontinuously at certain endogenous spot price processes does not disappear in continuous-time; it only becomes more difficult to deal with.) In the single-commodity setting of this paper, however, one can define spanning assumptions directly on a real risky security dividend process $Y=\left(Y^{1}, \ldots, Y^{N}\right)$, provided there is also a nominal numeraire security $D^{0}$ as defined in Section 3 . In this case, the primitives of an economy are $\hat{\mathscr{E}}=\left((\Omega, \mathscr{F}, F, P),\left(U_{i}, e^{i}\right),\left(Y, D^{0}\right)\right)$.

Suppose, for $N \geqslant K$, that $Y$ is an $\mathbf{R}^{N}$-valued Ito process with stochastic differential representation

$$
d Y_{t}=\mu_{Y}(t) d t+\sigma_{Y}(t) d B_{t},
$$

and that $M_{t}=\int_{0}^{t} \sigma_{Y}(s) d B_{s}$ is a martingale generator (as defined in (A.3)). For this, it is basically enough that the essential infimum of the rank of $\sigma_{Y}$ is maximal, and therefore equal to the dimension $K$ of the Brownian motion $B$. As an alternative to (A.3), this is in principle enough to demonstrate the existence of an equilibrium satisfying all of the results of the paper. To guarantee this, we need only complete the proof of Step (C) of Theorem 1, as follows.

We take static equilibrium $\left(x^{1}, \ldots, x^{m} ; \pi\right)$ as given by Appendix $\mathrm{A}$, Theorem A4, where $\pi$ has the representation $\pi \cdot c=E\left(\int_{0}^{T} p_{t} c_{t} d t\right)$, for $p$ bounded and bounded away from zero. The corresponding nominal dividend process $D^{n}$ is defined by $d D_{t}^{n}=p_{t} d Y_{t}^{n}$. By Girsanov's Theorem (under technical regularity conditions on $\mu_{Y}$ and $\sigma_{Y}(t)$ given in Lipster and Shiryayev (1977)), there exists a new probability measure $Q$ uniformly equivalent to $P$ and a Brownian motion $\hat{B}$ relative to $Q$ under which $d D_{t}=p_{t} \sigma_{Y}(t) d \hat{B}_{t}$. Since $p$ is bounded above and below away from zero, the spanning assumption (A.3) is therefore satisfied under the new measure $Q$. Let $\tilde{S}_{t}=E^{Q}\left[D_{T}-D_{t} \mid \mathscr{F}_{t}\right]$ define the nominal security price processes, where $E^{Q}$ denotes expectation under $Q$. In order to define a suitable 
new consumption spot price process $\tilde{p}$, first define the density process

$$
z_{t}=E\left[\frac{d Q}{d P} \mid \mathscr{F}_{t}\right], \quad t \in[0, T] .
$$

Now let $\tilde{p}_{t}=p_{t} / z_{t}$ for all $t$. By Lemma 4.1 and Proposition 4.1 of Duffie (1986), there exist trading strategies $\left(\tilde{\theta}^{1}, \ldots, \tilde{\theta}^{m}\right)$ such that $\left((\tilde{S}, \tilde{p}),\left(x^{1}, \tilde{\theta}^{1}\right), \ldots,\left(x^{m}, \tilde{\theta}^{m}\right)\right)$ is an equilibrium for $\hat{\mathscr{E}}$. In order to recover the pricing formula (6) on which the rest of the paper is based, we use the fact that, for any integrable random variable $W$,

$$
E^{Q}\left(W \mid \mathscr{F}_{t}\right)=\frac{E^{P}\left(W z_{T} \mid \mathscr{F}_{t}\right)}{z_{t}} .
$$

Combining this rule with the arguments used in the proof of Lemma 4.1 of Duffie (1986), it is then easily shown that the real price process $\hat{S}$ defined by $\hat{S}_{t}=\tilde{S}_{t} / \tilde{p}_{t}$ satisfies the basic representative asset pricing equation (6), and the paper continues as before.

This Appendix shows that, in this special setting, one can actually provide conditions directly on real primitive security dividends that generate spanning in equilibrium. Aside from being somewhat more complicated and requiring additional technical regularity conditions, this yields the same asset pricing results stated in the body of the paper.

\section{REFERENCES}

ARRow, K. (1953): "Le rôle des valeurs boursières pour la repartition la meillure des risques," Econometrie. Colloques Internationaux Centre National de la Recherche Scientifique (Paris, 1952), 40, pp. 41-47; discussion, pp. 47-48. Paris: C.N.R.S., 1953. English translation: Review of Economic Studies, 31 (1964), 91-96.

Aliprantis, C., D. Brown, AND O. Burkinshaw (1987a): “Edgeworth Equilibria," Econometrica, $55,1109-1137$.

(1987b): "Edgeworth Equilibria in Production Economies," Journal of Economic Theory, 43, 252-291.

Araujo, A., AND P. Monteiro (1986): “Existence Without Uniform Conditions," Unpublished, IMPA, Rio de Janeiro.

(1987): "Generic Non-existence of Equilibria in Finance Models," Unpublished, IMPA, Rio de Janeiro.

BACK, K. (1988): “Correlation of Consumption, State Prices, and Risk Premia for General Asset Price Processes," Unpublished, University of Indiana.

Bewley, T. (1972): "Existence of Equilibria in Economies with Infinitely Many Commodities," Journal of Economic Theory, 4, 514-540.

Breeden, D. (1979): “An Intertemporal Asset Pricing Model with Stochastic Consumption and Investment Opportunities," Journal of Financial Economics, 7, 265-296.

Breeden, D., M. GibBons, AND B. Litzenberger (1986): "Empirical Tests of the Consumption-Oriented CAPM," Research Paper 879, Graduate School of Business, Stanford University.

BRown, D. (1983): "Existence of Equilibria in a Banach Lattice with Order Continuous Norm," Unpublished, Cowles Foundation, Yale University.

Chamberlain, G. (1988): “Asset Pricing in Multiperiod Securities Markets," Econometrica, 56, 1283-1300.

Cox, J., J. INGERsoll, AND S. Ross (1985): “An Intertemporal General Equilibrium Model of Asset Prices," Econometrica, 53, 363-384.

Dana, R.-A., AND M. Pontier (1989): “On the Existence of a Stochastic Equilibrium. A Remark," Unpublished, Laboratoire de Mathématiques Fondamentales, Université de Paris VI; Département de Mathématiques et d'Informatique, Université d'Orleans.

DufFIE, D. (1986): "Stochastic Equilibria: Existence, Spanning Number, and The 'No Expected Financial Gain From Trade' Hypothesis," Econometrica, 54, 1161-1184.

DufFIE, D., AND C. HuANG (1985): "Implementing Arrow-Debreu Equilibria by Continuous Trading of Few Long-Lived Securities," Econometrica, 53, 1337-1356.

Epstein, L., AND S. ZIN (1989): "Substitution, Risk Aversion and the Temporal Behavior of Consumption and Asset Returns: A Theoretical Framework," Unpublished, Department of Economics, University of Toronto, forthcoming in Econometrica. 
Grossman, S., and G. Laroque (1987): “Asset Pricing and Optimal Portfolio Choice in the Presence of Illiquid Durable Consumption Goods," Unpublished, Princeton University.

HART, O., (1975): “On the Optimality of Equilibrium when the Market Structure is Incomplete," Journal of Economic Theory, 11, 418-443.

HuANG, C. (1987): "An Intertemporal General Equilibrium Asset Pricing Model: The Case of Diffusion Information," Econometrica, 55, 117-142.

Karatzas, I., P. Lakner, J. P. Lehoczky, and S. Shreve (1988): "Dynamic Equilibrium in a Multi-Agent Economy: Construction and Uniqueness," Research Paper 88-9, Department of Mathematics, Carnegie-Mellon University.

LehoczKy, J. P., AND S. Shreve (1986): "Explicit Equilibrium Solutions for a Multi-Agent Consumption/Investment Problem," Technical Report 384, Department of Statistics, Carnegie-Mellon University.

LinTNER, J. (1965): “The Valuation of Risky Assets and the Selection of Risky Investment in Stock Portfolios and Capital Budgets," Review of Economics and Statistics, 47, 13-37.

LipSTer, R. Sh., AND A. N. ShiryaYeV (1977): Statistics of Random Processes. New York, Berlin: Springer-Verlag.

LUCAS, R. (1978): “Asset Prices in an Exchange Economy,” Econometrica, 46, 1429-1445.

Mas-Colell, A. (1986): “The Price Equilibrium Existence Problem in Topological Vector Lattices," Econometrica, 54, 1039-1054.

Merton, R. (1973): “An Intertemporal Capital Asset Pricing Model,” Econometrica, 41, 867-888.

$\rightarrow$ Rubinstein, M. (1976): "The Valuation of Uncertain Income Streams and the Pricing of Options," Bell Journal of Economics, 7, 407-425.

SCHAEFER, H. (1974): Banach Lattices and Positive Operators. Berlin: Springer-Verlag.

Sharpe, W. (1964): "Capital Asset Prices: A Theory of Market Equilibrium Under Conditions of Risk," Journal of Finance, 19, 425-442.

YANNELIS, N., AND W. ZAME (1986): "Equilibria in Banach Lattices Without Ordered Preferences," Journal of Mathematical Economics, 15, 85-110.

ZAME, W. (1987): "Competitive Equilibria in Production Economies with an Infinite-Dimensional Commodity Space,” Econometrica, 55, 1075-1108. 